\title{
A fluctuating elastic plate and a cell model for lipid membranes
}

\author{
Xiaojun Liang and Prashant K. Purohit* \\ Department of Mechanical Engineering and Applied Mechanics \\ University of Pennsylvania \\ Philadelphia, PA 19104
}

\begin{abstract}
The thermal fluctuations of lipid bi-layer membranes are key to their interaction with cellular components as well as the measurement of their mechanical properties. Typically, membrane fluctuations are analyzed by decomposing into normal modes or by molecular simulations. Here we propose two new approaches to calculate the partition function of a membrane. In the first approach we view the membrane as a fluctuating von Karman plate and discretize it into triangular elements. We express its energy as a function of nodal displacements, and then compute the partition function and co-variance matrix using Gaussian integrals. We recover well-known results for the dependence of the projected area of the membrane on the applied tension and recent simulation results on the dependence of membrane free energy on geometry, spontaneous curvature and tension. As new applications we compute the fluctuations of the membrane of a malaria infected cell and analyze the effects of boundary conditions on fluctuations. Our second approach is based on the cell model of Lennard-Jones and Devonshire. This model, which was developed for liquids, assumes that each molecule fluctuates within a cell on which a potential is imposed by all the surrounding molecules. We adapt the cell model to a lipid membrane by recognizing that it is a 2D liquid with the ability to deform out of plane whose energetic penalty must be factored into the partition function of a cell. We show, once again, that some results on membrane fluctuations can be recovered using this new cell model. However, unlike some well established results, our cell model gives an entropy that scales with the number of molecules in a membrane. Our model makes predictions about the heat capacity of the membrane that can be tested in experiments.
\end{abstract}

Keywords: Membrane Thermodynamics, Finite Element Computation, Cell Model

\section{Introduction}

Thermal fluctuations of two-dimensional surfaces have been studied since at least the 1970s [1, 2]. At that time analytic and experimental methods to study fluctuations were applied to liquid-vapor interfaces and lipid bi-layers.

${ }^{*}$ Corresponding author.

Tel: (215)8983870

Fax: (215)5736334

Email: purohit@seas.upenn.edu

Preprint submitted to Elsevier

January 26, 2016 
The same methods are now used to extract the mechanical properties of two-dimensional solids, such as, graphene [3] and polymeric membranes [4]. It is appropriate to think of these two-dimensional solids as fluctuating elastic plates since they resist bending. However, the term 'membrane' is more commonly used in the literature for these objects. In this paper we will use 'plate' and 'membrane' interchangeably even though in mechanics membranes are understood to be objects that have no bending rigidity. Experiments [5] show that the response of a membrane under two-dimensional hydro-static tension has two regions. In the low tension region, the projected area of the membrane increases logarithmically with increasing tension due to the stretching out of thermal fluctuations. In the high tension region, the projected area increases linearly with increasing tension. In this linear region fluctuations have already been stretched out and the increase in area is due to the increase in the inter-molecular distance. This is very much like the increase in volume of a solid under hydro-static tension and is characterized by an area expansion modulus which is the two-dimensional analogue of the bulk modulus $[5,8]$. The low tension region is dominated by entropy and is the main focus of our study.

Many previous analytic studies of the thermal fluctuation of membranes relied on Fourier series (or series expansions in other orthogonal bases) $[3,6,7,8,9,10,11,12]$ to decompose the membrane fluctuation into normal modes. Then the mean square amplitude of each mode was obtained using the equipartition theorem. Finally, integration over all wave-lengths lead to an expression for the change in projected area of the membrane as a function of the tension. However, these treatments did not examine the role of boundary conditions on membrane fluctuations. For example, we expect smaller fluctuations if we apply clamped-clamped boundary conditions on a fluctuating plate than when we apply simply supported boundary conditions.

Membrane statistical mechanics has also been studied using different simulation methods. In these studies the membrane is first discretized and its energy is written in terms of the discretized variables. Fluctuations are then analyzed using Monte Carlo techniques [13, 14, 15, 16, 17, 18, 19, 20]. Unlike the Fourier series approaches, Monte Carlo methods are not restricted to small fluctuations of membranes. They are general enough to account for the coupling of out-of-plane displacement to in-plane strains [3, 22], as well as different types of boundary conditions. Using Monte Carlo simulations the effects of proteins on membranes have also been studied [15, 19, 21]. Another class of simulation methods that has been used to study membrane fluctuations is the time dependent Ginzburg-Landau (TDGL) method. The advantage of these methods is that they can capture the temporal response of membranes $[23,24]$ while Monte Carlo methods give only the equilibrium response. However, it is time consuming to compute membrane free energies using Monte Carlo or TDGL methods. We refer the reader to a review by Ramkrishna et al. [21] for a detailed description of the state of the art in simulation methods for membranes.

Here we propose a new approach to compute membrane free energies and fluctuations. We discretize a membrane using equilateral triangle elements [25]. Then we express the energy of the membrane in terms of displacements of each node exactly as in finite elements, like many Monte Carlo and other simulations do [26, 27]. However, we compute the partition function of the membrane using a different strategy based on Gaussian integrals [28, 29, 30] that has been successfully applied to fluctuating elastic rods[31, 32] and fiber networks[33, 34]. This has the advantage 
that it involves lesser computational effort than Monte Carlo or TDGL methods if the size of the membrane is not very large. The disadvantage is that we are confined to fluctuations that are not so large as to cause overhangs in the membrane configuration (this is similar to the Monge gauge [8]) resulting in non-quadratic Hamiltonians. Using our method we first recover some known results on membrane fluctuations. Then, we show how different boundary conditions can affect the fluctuations. We also use our method to compute free energies of a membrane with regions of different spontaneous curvature. Our method allows us to study heterogeneous membranes which are the norm in biological cells. We apply it to a recent experiment on the fluctuations of cell membranes infected with malarial parasite [35].

Towards the end of the paper we also present a different method to compute the partition function of a membrane following the classic work of Lennard-Jones and Devonshire [36, 37]. The 'cell model' of these authors has been shown to capture the thermodynamic properties of inert gas liquids [38]. The key assumption of this model is that each molecule of the liquid is free to fluctuate inside a cell on which a potential is imposed by all the other molecules surrounding it. A lipid bi-layer is a liquid in-plane, yet the cell model has not been invoked to describe its fluctuations, even though suggestions to this effect can be found in the literature [39]. The cell model has been applied to study the interactions of proteins embedded in lipid bi-layer membranes [40, 41, 42]. In those models the size of the cell is determined by the concentration of the proteins on the membrane. In our case there is no protein, so the size of the cells is determined by the tension and the bending modulus. Our model is reminiscent of the freely-jointed-chain model for polymers in which the partition function $Z$ of the chain can be written as $Z=z^{n}$, where $z$ is the partition function of a single link and $n$ is the number of links in the chain. A model for the partition function of a confined lipid membrane that had the same form was given by Freund [43].

\section{Fluctuating elastic plate}

\section{1. von Karman energy}

Consider a thin square membrane (or plate) of side $L$ with a finite bending modulus $K_{b}$ everywhere as shown in Figure 1. Here we assume that $K_{b}$ is a constant everywhere, but later in the paper we allow $K_{b}$ to be a function of reference coordinates $x$ and $y$. We assume that the plate is under tension, but due to thermal fluctuations it can suffer moderately large deflections. According to the von Karman plate theory, the elastic energy $E$ of such a plate can be expressed as a combination of the stretching and bending energies as in [44]:

$$
\begin{aligned}
E=E_{s}+E_{b} & =\frac{Y h}{2\left(1-v^{2}\right)} \iint \mathrm{d} x \mathrm{~d} y\left[\left(\varepsilon_{x}+\varepsilon_{y}\right)^{2}-2(1-v)\left(\varepsilon_{x} \varepsilon_{y}-\varepsilon_{x y}^{2}\right)\right] \\
& +\frac{Y h^{3}}{24\left(1-v^{2}\right)} \iint \mathrm{d} x \mathrm{~d} y\left[\left(w_{, x x}+w_{, y y}\right)^{2}-2(1-v)\left(w_{, x x} w_{, y y}-w_{, x y}^{2}\right)\right],
\end{aligned}
$$

where $Y$ and $v$ are the Young's modulus and Poisson ratio of the material respectively, and $h$ is the thickness of the membrane. The variables $\varepsilon_{x}, \varepsilon_{y}, \varepsilon_{x y}$, and $w$ in the integral are respectively the in-plane strains and out-plane 


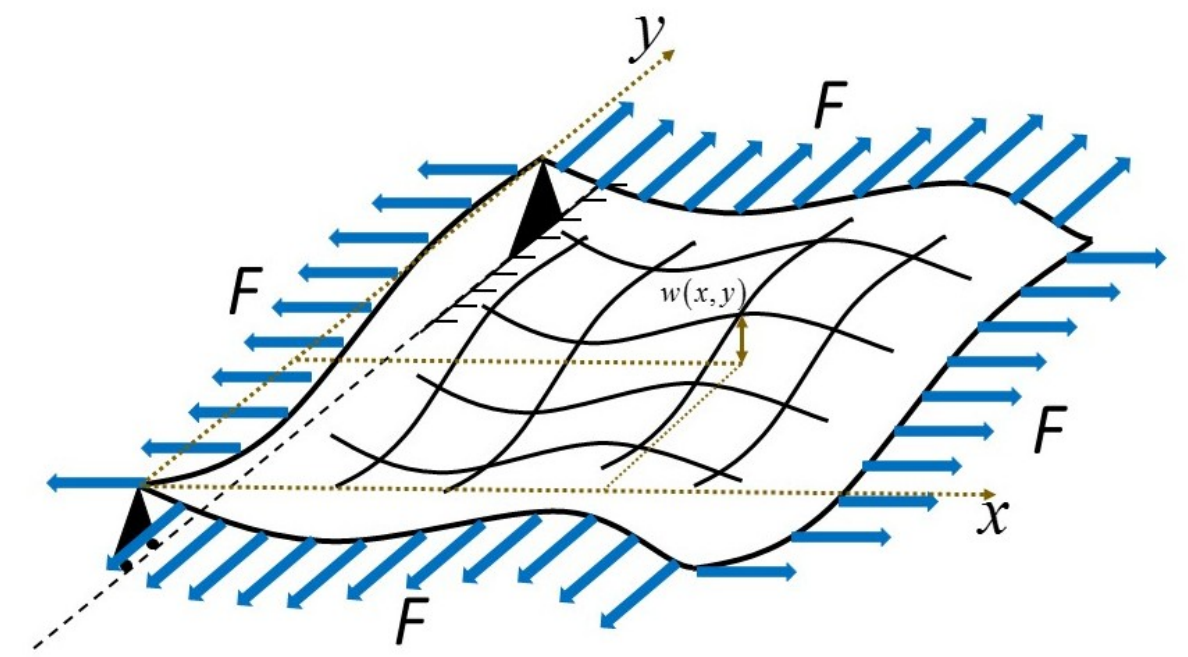

Figure 1: Fluctuating elastic plate with applied hydro-static tension $F$. The out-of-plane deflection of reference point $(x, y)$ is $w(x, y)$.

displacement of the neutral plane. The sub index ${ }_{, x x}, x y$, , yy represent second derivatives respectively with respect to the reference coordinates. von Karman also deduced the geometrical relation that links the in-plane strains to $w(x, y)$ and the two in-plane displacements $u, v$ respectively in the $x$ and $y$ directions. This relation is summarized by

$$
\begin{aligned}
\varepsilon_{x} & =u_{, x}+\frac{w_{, x}^{2}}{2}, \\
\varepsilon_{y} & =v_{, y}+\frac{w_{, y}^{2}}{2}, \\
\varepsilon_{x y} & =\frac{u_{, y}+v_{, x}}{2}+\frac{w_{, x} w_{, y}}{2} .
\end{aligned}
$$

The in-plane strains $\varepsilon_{x}, \varepsilon_{y}, \varepsilon_{x y}$ are related to the out-of-plane displacement $w(x, y)$ through a compatibility condition which involves the Gauss curvature of the membrane [44]. Now, consider applying a two-dimensional hydro-static tension of magnitude $F$ along all the edges of our fluctuating membrane, as shown in Figure 1. Then from Eqn. (2), the potential energy due to this tension is given by

$$
P E=-\iint F\left(u_{, x}+v_{, y}\right) \mathrm{d} x \mathrm{~d} y=-\iint F\left(\varepsilon_{x}-\frac{w_{, x}^{2}}{2}+\varepsilon_{y}-\frac{w_{, y}^{2}}{2}\right) \mathrm{d} x \mathrm{~d} y .
$$

Assuming two-dimensional plane stress, let us define $K=Y /(2-2 v)$ as the two-dimensional bulk modulus. We use $K$ and shear modulus $G=Y /(2+2 v)$ instead of Young's modulus $Y$ and Poisson's ratio $v$ as the two mechanical parameters for an isotropic material, then

$$
\frac{Y}{1-v^{2}}=\frac{1}{2}\left(\frac{Y}{1+v}+\frac{Y}{1-v}\right)=K+G .
$$


Using this we can rewrite the expression for the von-Karman energy as

$$
\begin{aligned}
& E_{s}=\iint \mathrm{d} x \mathrm{~d} y\left\{\frac{K h}{2}\left(\varepsilon_{x}+\varepsilon_{y}\right)^{2}+\frac{G h}{2}\left[\left(\varepsilon_{x}-\varepsilon_{y}\right)^{2}+\varepsilon_{x y}^{2}\right]\right\} \\
& E_{b}=\iint \mathrm{d} x \mathrm{~d} y\left\{\frac{K h^{3}}{24}\left(w_{, x x}+w_{, y y}\right)^{2}+\frac{G h^{3}}{24}\left[\left(w_{, x x}-w_{, y y}\right)^{2}+w_{, x y}^{2}\right]\right\} .
\end{aligned}
$$

Adding the potential energy to this expression and denoting $K h^{3} / 12$ as a new bending modulus $K_{b}$, we get the total energy of the fluctuating plate as $E=E_{s}+E_{b}$, where

$$
\begin{aligned}
& E_{s}=\iint \mathrm{d} x \mathrm{~d} y\left\{\frac{K h}{2}\left(\varepsilon_{x}+\varepsilon_{y}\right)^{2}+\frac{G h}{2}\left[\left(\varepsilon_{x}-\varepsilon_{y}\right)^{2}+\varepsilon_{x y}^{2}\right]-F\left(\varepsilon_{x}+\varepsilon_{y}\right)\right\}, \\
& E_{b}=\iint \mathrm{d} x \mathrm{~d} y\left\{\frac{K_{b}}{2}\left(w_{, x x}+w_{, y y}\right)^{2}+\frac{G h^{3}}{24}\left[\left(w_{, x x}-w_{, y y}\right)^{2}+w_{, x y}^{2}\right]+\frac{F}{2}\left(w_{, x}^{2}+w_{, y}^{2}\right)\right\} .
\end{aligned}
$$

We will use this energy to calculate the partition function $Z$ of our plate. But, in order to calculate the partition function we must first discretize our plate and express the energy in terms of the discretized variables.

\subsection{Discretization into triangular elements}

In this section we specialize to a liquid membrane and show how we discretize it to compute the energy. The in-plane shear modulus $G$ is assumed to be zero. Then the energy expressions reduce to

$$
\begin{aligned}
& E_{s}=\iint \mathrm{d} x \mathrm{~d} y\left\{\frac{K h}{2}\left(\varepsilon_{x}+\varepsilon_{y}\right)^{2}-F\left(\varepsilon_{x}+\varepsilon_{y}\right)\right\}, \\
& E_{b}=\iint \mathrm{d} x \mathrm{~d} y\left\{\frac{K_{b}}{2}\left(w_{, x x}+w_{, y y}\right)^{2}+\frac{F}{2}\left(w_{, x}^{2}+w_{, y}^{2}\right)\right\} .
\end{aligned}
$$

To further simplify the problem let us assume that our membrane is inextensible, or $\varepsilon_{x}+\varepsilon_{y}=0$ everywhere. Hence, the only terms left in the energy expression are the bending energy and potential energy of the applied tension $F$ caused by out-of-plane displacement of the membrane. For any given $\varepsilon_{x}, \varepsilon_{y}$ and $w$, we can choose an appropriate $\varepsilon_{x y}$ to satisfy the compatibility equation because in-plane shears do not cost any energy for a liquid plate. If the in-plane shear modulus was non-zero then this would not be the case and we would have to impose the compatibility condition as a constraint everywhere on the plate. For the discretized plate this would require computation of the Gauss curvature at every node. This point has been treated in detail in $[3,12]$.

We discretize the membrane into $Q \approx 4 N^{2} / \sqrt{3}$ equilateral triangles with $P \approx 2 N(N+1) / \sqrt{3}$ node points, where $N=L / l, L$ is the length of the membrane and $l$ is the side of each triangle. The discretization scheme is shown in Figure 2a. If the out-of-plane displacements at the nodes are known we assume that the displacements in the interior of the triangular element can be obtained by linear interpolation. Thus, our planar triangle element in the reference configuration goes to a planar triangle in the deformed configuration (see Figure 2a). Then we can discretize the total energy into the expression below as discussed in several papers [26, 45, 46]. 


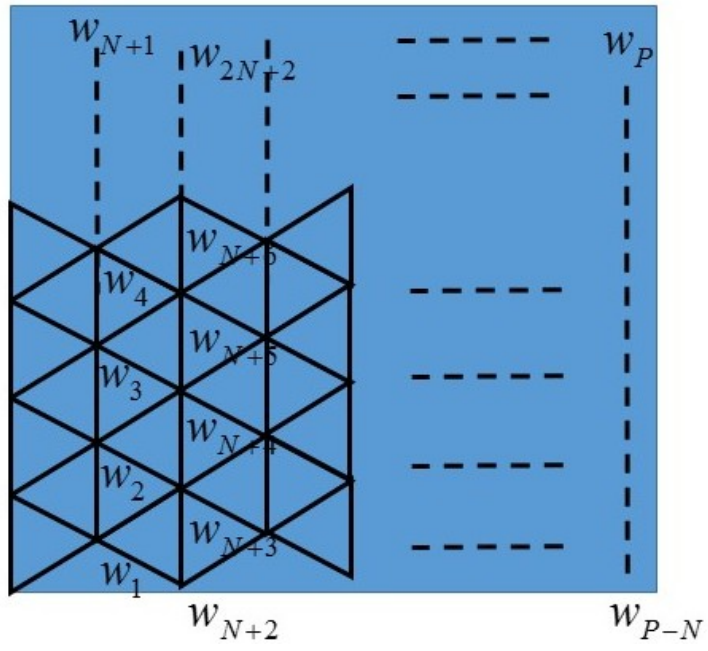

(a)

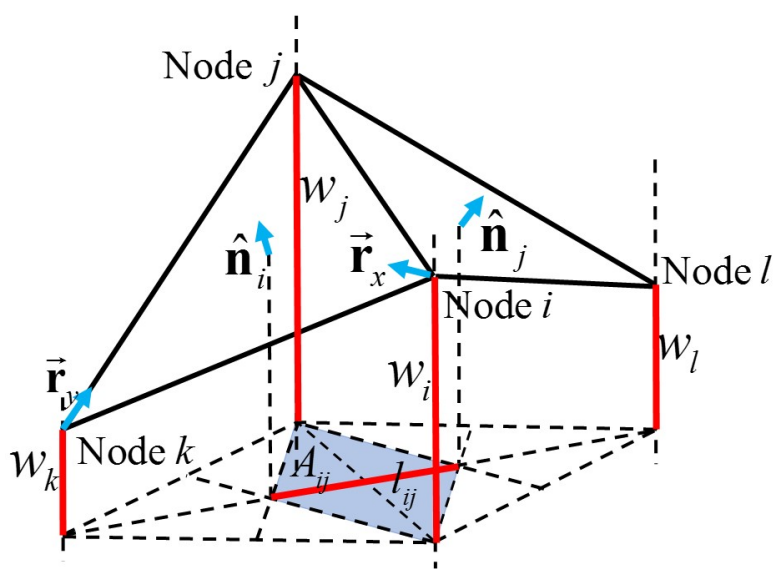

(b)

Figure 2: (a) Equilateral triangles discretization of a square plate. (b) Reference and deformed configuration of two adjacent elements. The reference configuration is drawn with dashed lines. Each triangular element deforms into a triangle in the deformed configuration.

$$
E=\frac{K_{b}}{2} \sum_{(i, j)} A_{i j}\left|\frac{\hat{n}_{i}-\hat{n}_{j}}{l_{i j}}\right|^{2}+\frac{F}{2} \sum A_{e}\left(w_{, x}^{2}+w_{, y}^{2}\right) .
$$

Here, the mean curvature term due to the out-of-plane displacement $w_{, x x}+w_{, y y}$ is expressed in terms of the difference of normal vectors $\hat{n}$ of the two adjacent triangle elements. The summation in the potential energy of the tension $F$ runs over all the triangle elements, and the summation in the bending energy term runs over all the adjacent triangle element pairs that share one edge linked by nodes $i, j . \quad l_{i j}$ is the length between the centers of these two triangle elements. $A_{e}=L^{2} / Q$ is the reference area of one triangle element, $A_{i j}$ is the area associated with edge $i j$, which equals $2 A_{e} / 3$ in the reference configuration. $l_{i j}$ and $A_{i j}$ are shown in Figure $2 \mathrm{~b}$ in which nodes $i, j, k, l$ form two elements sharing one edge linked by nodes $i, j . w_{, x}, w_{, y}$, and $\hat{n}$ for the element formed by nodes $i, j, k$ are given in terms of the three node displacements $w_{i}, w_{j}, w_{k}$ as

$$
\begin{aligned}
& w_{, x}=\frac{2 w_{i}-w_{j}-w_{k}}{\sqrt{3} l}, w_{, y}=\frac{w_{j}-w_{k}}{l}, \\
& \vec{r}_{x}=\frac{\left(0,1, w_{, x}\right)}{\sqrt{1+w_{, x}^{2}}}, \quad \vec{r}_{y}=\frac{\left(0,1, w_{, y}\right)}{\sqrt{1+w_{, y}^{2}}}, \\
& \hat{n}_{i}=\vec{r}_{x} \times \vec{r}_{y}=\frac{\left(-w_{, x},-w_{, y}, 1\right)}{\sqrt{1+w_{, x}^{2}+w_{, y}^{2}+w_{, x}^{2} w_{, y}^{2}}} .
\end{aligned}
$$

Similarly $w_{, x}, w_{, y}$, and $\hat{n}$ for the element formed by nodes $i, j, l$ are given in terms of the three node displacements $w_{i}, w_{j}, w_{l} \cdot w_{, x}^{2}$ and $w_{, y}^{2}$ are assumed small enough that the denominator in the expression for $\hat{n}_{i}$ can be expanded in a 
Taylor series. However, if we retain terms only upto quadratic order in $w(x, y)$ in the energy then $w_{, x}^{2}$ and $w_{y}^{2}$ from the denominators do not contribute. Substituting the result into Eqn. (8) we get the energy expression as a quadratic function of the nodal displacements for the two elements shown in Figure $2 b$ as

$$
E=\frac{4 K_{b} A_{e}}{3 l^{4}}\left(w_{i}+w_{j}-w_{k}-w_{l}\right)^{2}+\frac{F A_{e}}{3 l^{2}}\left[\left(w_{i}-w_{j}\right)^{2}+\left(w_{j}-w_{k}\right)^{2}+\left(w_{k}-w_{i}\right)^{2}\right] .
$$

We can repeat this exercise for all the elements and get the energy as a quadratic function of $\left\{w_{t}\right\}, t=1,2, \ldots, P$, where $P$ is the number of nodes, viz., $E=\mathbf{w M w}^{\mathrm{T}}$, where the vector $\mathbf{w}=\left[w_{1}, w_{2}, \ldots, w_{P}\right]$. The matrix $\mathbf{M}$ is the stiffness matrix and it is a function of $K_{b}, F, L, l$. The probability of finding the membrane in any given configuration is proportional to $\exp \left(-E / k_{B} T\right)$, where $E$ is the energy of this configuration, $k_{B}$ is the Boltzmann constant and $T$ is the absolute temperature. The partition function $Z$ of the membrane is obtained by integration of $\exp \left(-E / k_{B} T\right)$ over all possible configurations. To carry out this integration efficiently, we first recognize that at zero temperature (absence of fluctuations) the lowest energy configuration of our membrane is flat, $w(x, y)=0$ for all $x, y$. When $T>0$ the membrane fluctuates around this flat configuration. Second, we recognize that configurations with large deviations from this flat shape are energetically costly and will make exponentially small contributions to the partition function. This allows us to change the limits of the integrals over $d w_{j}, j=1,2, \ldots, P$ to $\pm \infty$. Then, the integration over all configurations for the partition function becomes easy to carry out as given in $[47,31,32,33]$. The partition function of the membrane scales with square root of the determinant of matrix $\mathbf{M}$, or the square root of the product of all eigenvalues of $\mathbf{M}$ as

$$
Z=\sqrt{\frac{\left(2 \pi k_{B} T\right)^{P}}{\operatorname{det} \mathbf{M}}}
$$

\subsection{Thermodynamics of the membrane}

The Gibbs free energy $G(F, T)$ of the membrane is related to the partition function $Z$ as $G=-k_{B} T \ln Z$. This quantity can also be computed using Monte Carlo and TDGL methods [23] for a triangulated membrane whose energy was written as a quadratic form above with the stiffness matrix $\mathbf{M}$. The computational effort involved in calculating these free energies using Monte Carlo or TDGL simulation methods depends on the acceptable error tolerance. The smaller the error tolerance, the more Monte Carlo moves are required. In our method the error in computation of the free energy arises from the numerical computation of the determinant of $\mathbf{M}$. If the size of the matrix is not very large then the error in the computation of the determinant is limited only by machine accuracy. Of course, discretizing a membrane also leads to errors in computation of the thermodynamic properties. We quantify this error in the following. We also show a comparison of the free energy computed using our method to that obtained by the TDGL method in section 2.6.

We can compute the properties of the membrane by computing derivatives of the free energy. First, the reduction in projected area caused by thermal fluctuation is defined as $\Delta A=A(\infty, T)-A(F, T)$, where $A(\infty, T)$ is the membrane area at very large tension such that all the undulations are stretched out and it is flat. The area reduction is conjugate to 
the tension $F$ in the free energy of the membrane, and can be computed as $\Delta A=-\partial G / \partial F$. Since all the terms related to the force $F$ in the expression for the partition function $Z$ are included in the matrix $\mathbf{M}$, we have

$$
\Delta A=-\frac{k_{B} T}{2} \frac{\mathrm{d}}{\mathrm{d} F} \ln (\operatorname{det} \mathbf{M})
$$

Second, the entropy is given by

$$
S=-\frac{\partial G}{\partial T}=\frac{k_{B}}{2}\left[P \ln \left(2 \pi k_{B} T\right)-\operatorname{det} \mathbf{M}+1\right]
$$

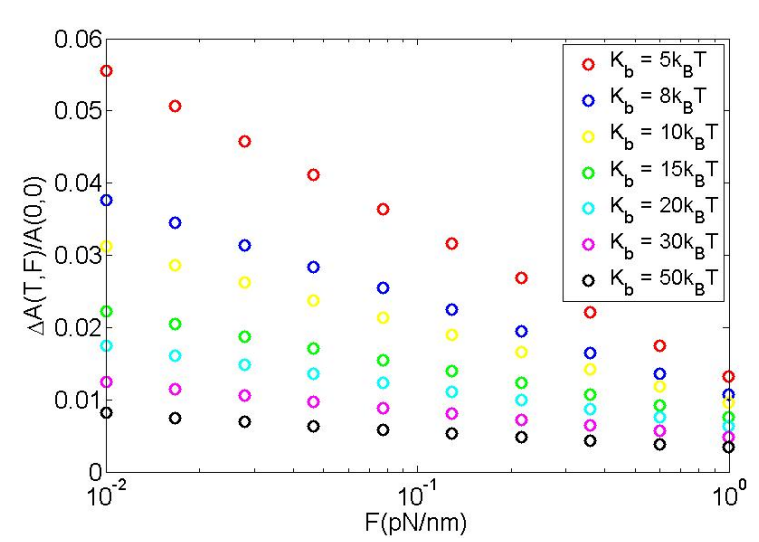

(a)

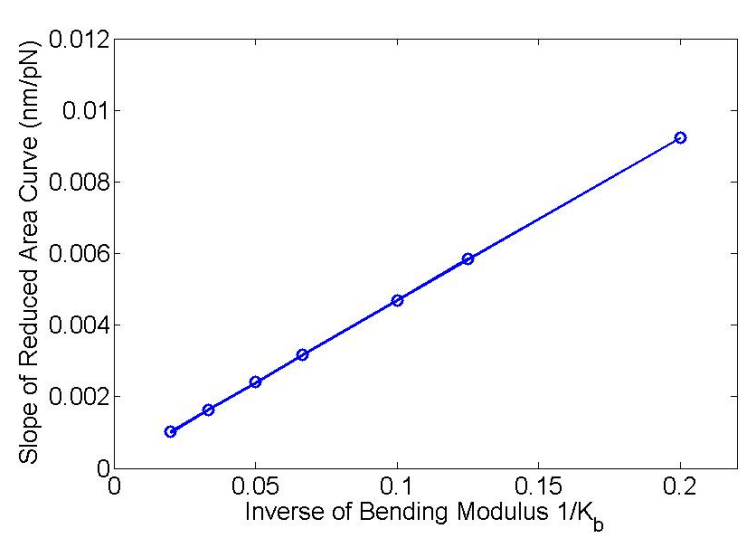

(b)

Figure 3: (a) Reduced area varies varies linearly as a function of $\log F$ for different values of bending modulus $K_{b}$. (b) Slopes of curves in (a) vary linearly with the inverse of bending modulus $K_{b}$.

We want to compare the results from these expressions with those expected from the Helfrich theory. In a typical membrane fluctuation problem in biology the size of the membrane is in microns, bending modulus of the membrane is around $10 k_{B} T$ and the typical tension range is from $0.01 \mathrm{pN} / \mathrm{nm}$ to $1 \mathrm{pN} / \mathrm{nm}$. So, for our computation, we consider a square patch of membrane of side $L=1 \mu \mathrm{m}$. Let us first examine the dependence of the reduced area $\triangle A / A$ obtained from our computations on the bending modulus $K_{b}$. Here $A=L^{2}$ is the reference area of the membrane at zero temperature. We compute the derivatives in Eqn. (12) for distinct values of membrane bending modulus and plot the results in Figure 3a. The slope of the curves (in the $\Delta A / A$ versus $\log F$ plot) varies linearly with the inverse of bending modulus $1 / K_{b}$ as shown in Figure $3 \mathrm{~b}$. This is consistent with the analytic solution given in $[6,7,8]$ and reproduced below:

$$
\frac{\Delta A}{A}=\frac{k_{B} T}{8 \pi K_{b}} \ln \frac{\pi^{2} / b^{2}+F / K_{b}}{\pi^{2} / A+F / K_{b}},
$$

where $b$ is the radius of a lipid head group and is on the order of $1 \mathrm{~nm}$. To compute the entropy of the membrane recall the Maxwell relation linking the projected area and the entropy:

$$
\left(\frac{\partial S}{\partial F}\right)_{T}=-\left(\frac{\partial \Delta A}{\partial T}\right)_{F}
$$


The right hand side of the above equation can be computed from (14). Then, by integration we get

$$
S(F, T)=\int-\left(\frac{\partial \Delta A}{\partial T}\right)_{F} \mathrm{~d} F=\frac{A k_{B}}{8 \pi}\left(\frac{\pi^{2}}{A}+\frac{F}{K_{b}}\right) \ln \left(\frac{\pi^{2}}{A}+\frac{F}{K_{b}}\right)-\frac{A k_{B}}{8 \pi}\left(\frac{\pi^{2}}{b^{2}}+\frac{F}{K_{b}}\right) \ln \left(\frac{\pi^{2}}{b^{2}}+\frac{F}{K_{b}}\right)+S_{0}(T)
$$

where $S_{0}(T)$ is some function of temperature $T$ and independent of membrane tension $F$.

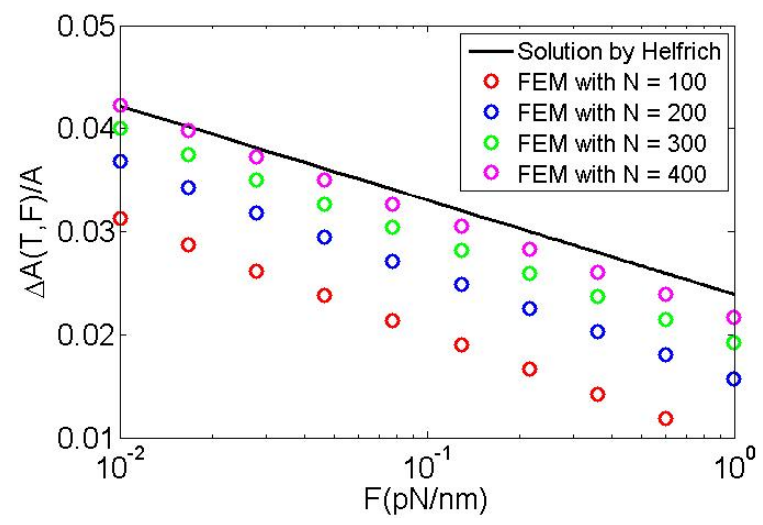

(a)

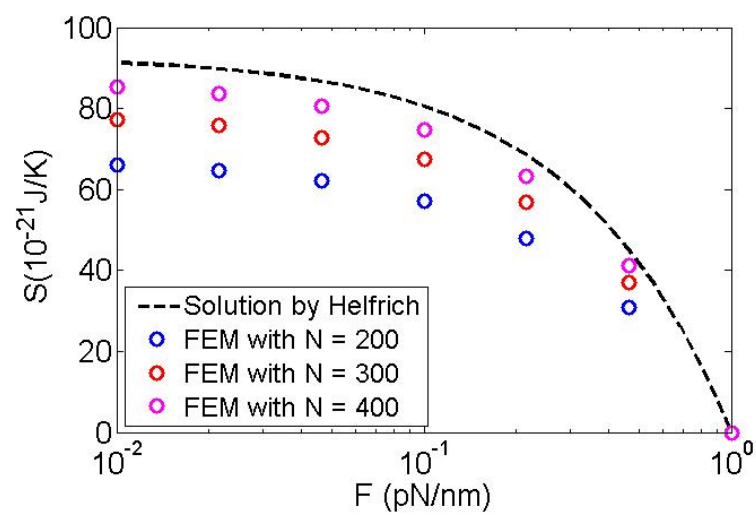

(b)

Figure 4: (a) Thermally reduced area computed with increasing number of elements with $K_{b}=10 k_{B} T$ at $T=300 \mathrm{~K}$. (b) Entropy change $\Delta S=S-S_{0}$ computed with increasing number of elements $N$. Notice that our numerical calculation based on (11) approaches the analytic solution of Helfrich as $N$ increases.

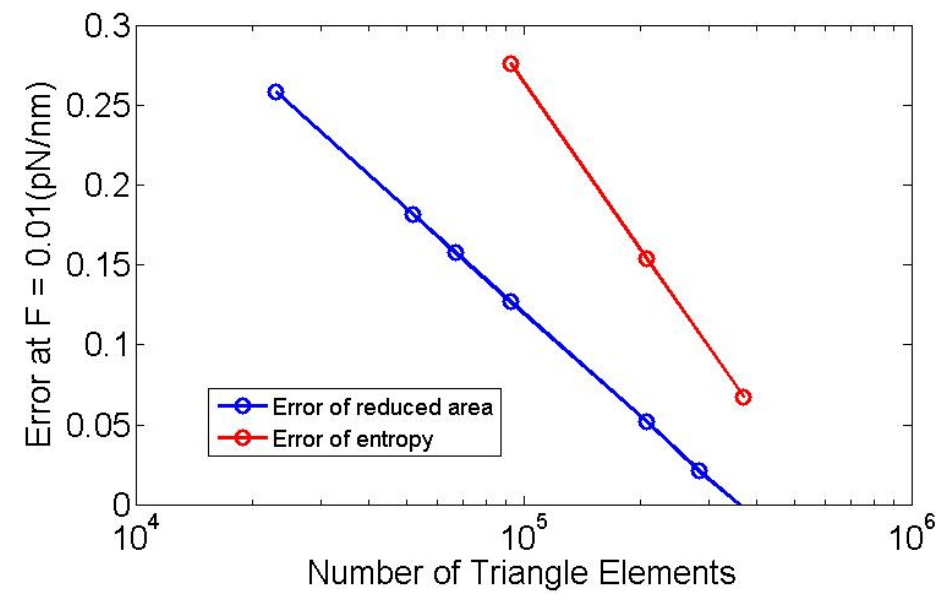

Figure 5: The error of our computational result, both for reduced area and entropy change, with respect to the Helfrich solution at $F=0.01 \mathrm{pN} / \mathrm{nm}$ decreases linearly with the logarithm of the number of elements.

In order to compare the results of our discretized model with this equation we change $N$ to see how small our elements should be in order for our curves to coincide with the analytic solution. We go as far as $N=400$ due to limitations of computational power. Note that the side of one equilateral triangle element at $N=400$ is about $2.5 \mathrm{~nm}$ since the side of our square membrane is 1 micron. This means that each element contains only about 10 lipids when 
$N=400$. The results of our computation are plotted in Figure $4 \mathrm{a}$ and $4 \mathrm{~b}$, respectively for reduced area $\Delta A / A$ and entropy change $\Delta S$. We see that our semi-analytic method approaches Eqns. (14) and (16) as $N$ increases. We can define an error by computing the distance between the analytic solution and the curve from our numerical computation for both the reduced area and the entropy. The error at tension $F=0.01 \mathrm{pN} / \mathrm{nm}$ decreases linearly with the logarithm of the number of elements (i.e. it decreases slowly) as shown in Figure. 5.

\subsection{Analysis of variance and effect of boundary conditions}

One important result which follows from the calculation of the partition function is the co-variance matrix $\left\langle w_{i} w_{j}\right\rangle$ $[47,31,32,33]$, which can be calculated from the inverse of the stiffness matrix $\mathbf{M}$ as

$$
\left\langle w_{i} w_{j}\right\rangle=\frac{k_{B} T}{\left[\mathbf{M}^{-1}\right]_{i j}} .
$$

From this we can get the variance of the out-plane deflection $\left\langle w_{i}^{2}\right\rangle$ as a function of reference position on our membrane. We have done this in Figure 6 for two different boundary conditions. Due to limitations computational power, we turn to a smaller $0.5 \mu \mathrm{m} \times 0.5 \mu \mathrm{m}$ membrane in order to keep the triangle element size $l=2.5 \mathrm{~nm}$ as in the previous computation of reduced area. In the first case one edge of the membrane is simply supported with the three other edges being free, while in the second case two opposite edges of the membrane are simply supported with the other two being free. The distance between the simply supported edges is allowed to change. In both cases a hydro-static tension $F$ per unit length is applied on the edges. In the first case the fluctuation increases as we go nearer the free boundaries, while in the second the fluctuation is maximum near the center of the membrane. In both cases the height fluctuation is on the order of several $\mathrm{nm}$ for given membrane properties and tension $F=0.01 \mathrm{pN} / \mathrm{nm}$. Most studies of membrane fluctuation do not account for the boundary conditions as we have done here. This could have implications on the interpretations of experiments which extract membrane properties by measuring thermal fluctuations.

\subsection{Persistence length}

Fluctuating polymers are often characterized by their persistence length $\xi_{p}$ [8]. This concept has been extended also to membranes. Roughly speaking, the persistence length is a length scale at which the thermal energy is comparable to the energy stored in elastic deformations. In other words, if the size of the elements is much larger than the persistence length the approximation of smooth deformations will be violated because shape fluctuations caused by thermal motion will obliterate the smoothness. Persistence lengths of lipid membranes are known to be much longer than the molecular scale [8]. One arrives at this conclusion by defining the persistence length $\xi_{p}$ through the decay in correlation of normal vectors of the membrane as

$$
\langle\mathbf{n}(0) \cdot \mathbf{n}(\Delta \mathbf{r})\rangle=\exp \left(-\frac{\Delta r}{\xi_{p}}\right)
$$




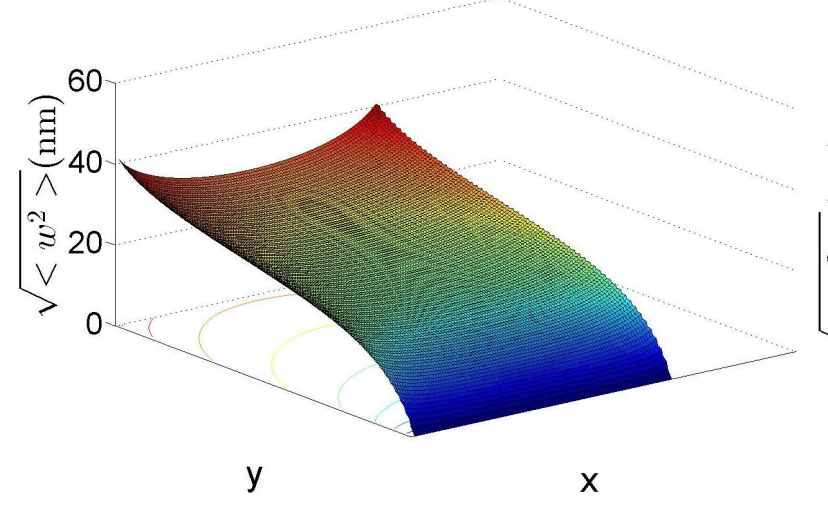

(a)

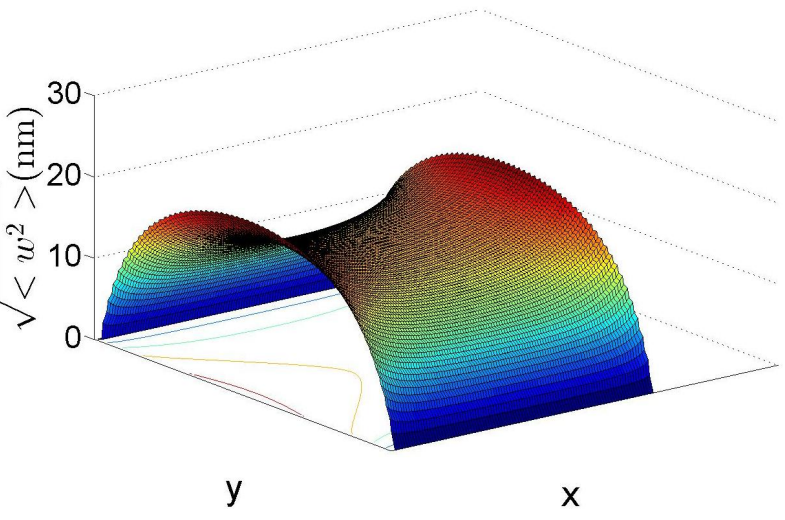

(b)

Figure 6: (a) Standard deviation in $w$ of a one end hinged membrane with tension $F=0.01 \mathrm{pN} / \mathrm{nm}$. (b) Standard deviation in $w$ of a two end hinged membrane with tension $F=0.01 \mathrm{pN} / \mathrm{nm}$. The magnitude of the fluctuation depends on the boundary conditions.

where $\Delta r$ is a distance coordinate. According to the derivation in [8], the correlation function can be reduced to

$$
\langle\mathbf{n}(0) \cdot \mathbf{n}(\Delta \mathbf{r})\rangle=1-\frac{k_{B} T}{2 \pi} \int \frac{I_{\theta}(z) q \mathrm{~d} q}{F+K_{b} q^{2}}
$$

where $z=q|\Delta \mathbf{r}|$ and

$$
I_{\theta}(z)=1-\frac{\int_{0}^{2 \pi} \mathrm{d} \theta \cos (z \cos \theta)}{2 \pi}
$$

is a Bessel function. At large $z$ the second term on the right hand side of the above equation is very close to 0 , so it is ignored in Eqn. (19). Hence, with $q$ ranging approximately from $\pi / \Delta r$ to $\pi / b$, where $b$ is the size of lipid head group, Eqn. (19) becomes:

$$
\langle\mathbf{n}(0) \cdot \mathbf{n}(\Delta \mathbf{r})\rangle \sim 1-\left.\frac{k_{B} T}{4 \pi K_{b}} \ln \left(F+K_{b} q^{2}\right)\right|_{\pi / \Delta r} ^{\pi / b} \sim 1-\frac{k_{B} T}{4 \pi K_{b}} \ln \frac{F+K_{b} \pi^{2} / b^{2}}{F+K_{b} \pi^{2} / \Delta r^{2}}
$$

If we set membrane tension $F$ to zero, Eqn. (21) becomes the expression in [8]:

$$
\langle\mathbf{n}(0) \cdot \mathbf{n}(\Delta \mathbf{r})\rangle \sim 1-\frac{k_{B} T}{2 \pi K_{b}} \ln \frac{\Delta r}{b} .
$$

By comparing this expression with a linearized version of Eqn. (18) one concludes that the persistence length of a membrane is very long [8]. On the other hand, according to the expression for the normal vector $\mathbf{n}$ given in Eqn. (9) the quantity $\langle\mathbf{n}(0) \cdot \mathbf{n}(\Delta \mathbf{r})\rangle$ can be related to the covariance matrix given in Eqn. (17). Suppose we want to compute the correlation of normal vector between two equilateral triangle elements whose nodal displacements are denoted by 
$w_{a}, w_{b}, w_{c}$ and $w_{d}, w_{e}, w_{f}$ respectively according to Eqn. 9, then

$$
\mathbf{n}(0)=\left(-\frac{2 w_{b}-w_{a}-w_{c}}{\sqrt{3} l},-\frac{w_{c}-w_{a}}{l}, 1\right), \quad \mathbf{n}(\Delta \mathbf{r})=\left(-\frac{2 w_{e}-w_{d}-w_{f}}{\sqrt{3} l},-\frac{w_{f}-w_{d}}{l}, 1\right) .
$$

From this we see that the average quantity $\langle\mathbf{n}(0) \cdot \mathbf{n}(\Delta \mathbf{r})\rangle$ is a sum of several entries in the covariance matrix $\left\langle w_{i} w_{j}\right\rangle$ of Eqn. (9). Hence, we are in a position to compare the correlation of the normal vectors from our computations with Eqn. (22). This exercise is performed in Figure 7. We choose the center of membrane as the origin where $\mathbf{r}=\mathbf{0}$ and extend $\Delta \mathbf{r}$ towards four directions on the plane. The four different color points represent computational results for the four directions. The result from our discretized model matches the theoretical prediction Eqn. (22) very well in Figure 7 for $F=0$. Our method also captures the trend in the correlation expected from Eqn. (21) for finite tension $F=0.1 \mathrm{pN} / \mathrm{nm}$. The disagreement at large values of $\Delta r$ is due to (a) approximations in the derivation of Eqn. (21), (b) from the fact that the analytic expression does not account for boundary conditions as we do in our computation, and (c) our's is a discretized model. While this reinforces our computational method, it also shows that Eqn. (18) is not how the correlations of the normal decay on a membrane.

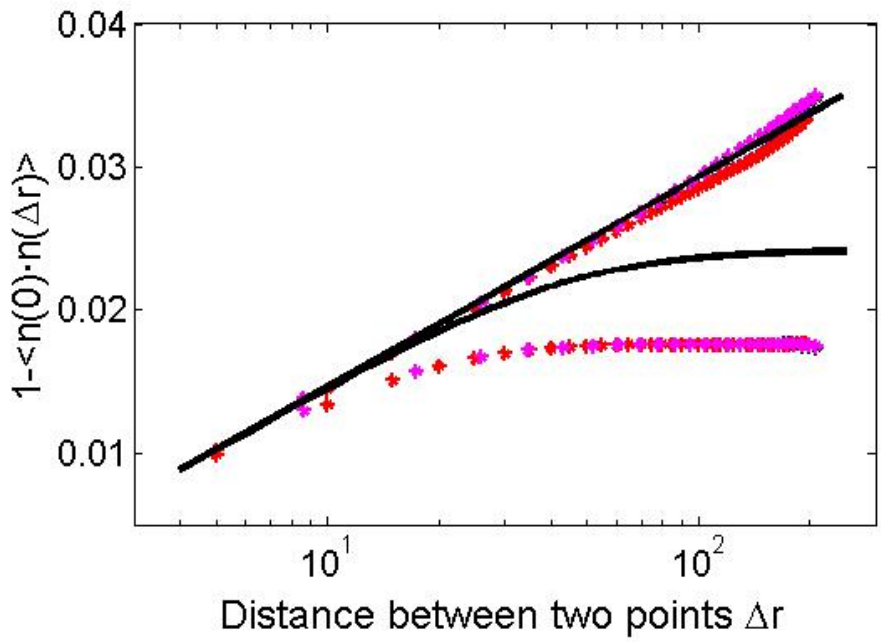

Figure 7: Change of correlation of normal vectors as functions of distance. We plot $1-\langle\mathbf{n}(0) \cdot \mathbf{n}(\Delta \mathbf{r})\rangle$ against the distance $\Delta r$. The straight black line is from Eqn. (21), and the curved black line is from Eqn. (22) for 0.1pN/nm tension. Blue, red, yellow, magenta lines are obtained from our computations and represent four distinct directions from the center of the membrane.

\subsection{Comparison to TDGL simulation}

An advantage of our method is that we can compute membrane free energies with modest computational effort. Here we compare our calculations with a TDGL simulation presented in [23]. In their calculation, a square patch of membrane is considered with non-zero spontaneous curvature $C_{0}$ in a circular region of radius $r_{0}$ in the center of the square. Their membrane is discretized by a 50-by-50 grid, and membrane free energy and entropy are obtained as a function of $r_{0}$ for different membrane sizes, bending modulus and tension. We will show that our model can be 
modified to account for spontaneous curvature and correctly obtain these quantities. To this end we add $C_{0}$ into Eqn. (8) for the energy as follows:

$$
E=\frac{K_{b}}{2} \sum_{(i, j)} A_{i j}\left(\left|\frac{\hat{n}_{i}-\hat{n}_{j}}{l_{i j}}\right|-C_{0}\right)^{2}+\frac{F}{2} \sum A_{e}\left(w_{, x}^{2}+w_{, y}^{2}\right) .
$$

Note that $C_{0}$ can be a function of position on the membrane. Now we follow the same procedure as we did for reaching Eqn. (10) and find that the energy takes the following form:

$$
E=\mathbf{w} \mathbf{M} \mathbf{w}^{\mathrm{T}}+\mathbf{w} \cdot \mathbf{b}+c
$$

where $\mathbf{b}$ is a vector that accounts for the non-zero spontaneous curvature associated with some node displacements $w_{i}$, and $c$ is a constant. Now, we differentiate Eqn. (25) with respect to $w_{i}, i=1,2, \ldots, P$ and set it to zero to get the equilibrium position $\bar{w}$ of the nodes as:

$$
\overline{\mathbf{w}}=-\frac{1}{2} \mathbf{M}^{-1} \mathbf{b}
$$

Let $\mathbf{w}=\overline{\mathbf{w}}+\delta \mathbf{w}$, where the new variable $\delta \mathbf{w}$ is a fluctuation around the equilibrium position. Therefore Eqn. (25) can be rewritten as:

$$
E=(\overline{\mathbf{w}}+\delta \mathbf{w}) \mathbf{M}(\overline{\mathbf{w}}+\delta \mathbf{w})^{\mathrm{T}}+(\overline{\mathbf{w}}+\delta \mathbf{w}) \cdot \mathbf{b}+c=\delta \mathbf{w} \mathbf{M} \delta \mathbf{w}^{\mathrm{T}}+\bar{c},
$$

where $\bar{c}=c+\frac{1}{2} \overline{\mathbf{w}} \cdot \mathbf{b}$ is independent of $\delta w$. Then, after factoring out $\bar{c}$ from the integration for the partition function, we get $[47,31,32,33]$ :

$$
Z=\exp \left(-\frac{\bar{c}}{k_{B} T}\right) \sqrt{\frac{\left(2 \pi k_{B} T\right)^{P}}{\operatorname{det} \mathbf{M}}} .
$$

To compare our computation with the TGDL simulation, we compute membrane free energy with exactly the same membrane properties and applied tension as in [23]. The side of the square patch is $250 \mathrm{~nm} . r_{0}$ is varied between 0 and $20 \mathrm{~nm}$, while $C_{0}$ is chosen to be $0.04 \mathrm{~nm}^{-1}$. The membrane is simply supported on four sides, and we use $N=300$. The CPU time for the computation of one curve in Figure 8a is less than 20 hours on a PC with a Intel(R) Core(TM) i7-3770 CPU @ 3.40GHz processor. Our result agrees with free energies obtained from the TDGL simulation as shown in Figure 8a. We also show a contour plot of equilibrium position as computed using Eqn. (26) in Figure 8b for zero tension. Our analysis shows that the spontaneous curvature does not affect the stiffness matrix M. Hence, the variance of the fluctuations given in Eqn. (17) is a function only of matrix $\mathbf{M}$ and is independent of the spontaneous curvature. The standard deviation of the fluctuations as a function of position can be seen in Figure $8 \mathrm{c}$ in which a cross-section of the membrane in equilibrium is depicted. Note also that spontaneous curvature does not change the entropy expression given in Eqn. (13). Therefore, in our calculation, change of magnitude or location of non-zero spontaneous curvature does not affect the entropy. This is also the case for the TDGL simulation in which every curve for entropy change (Figure 6 in [23]) is flat and near zero compared to the curve for free energy change due to different 
sizes of the non-zero spontaneous curvature region.

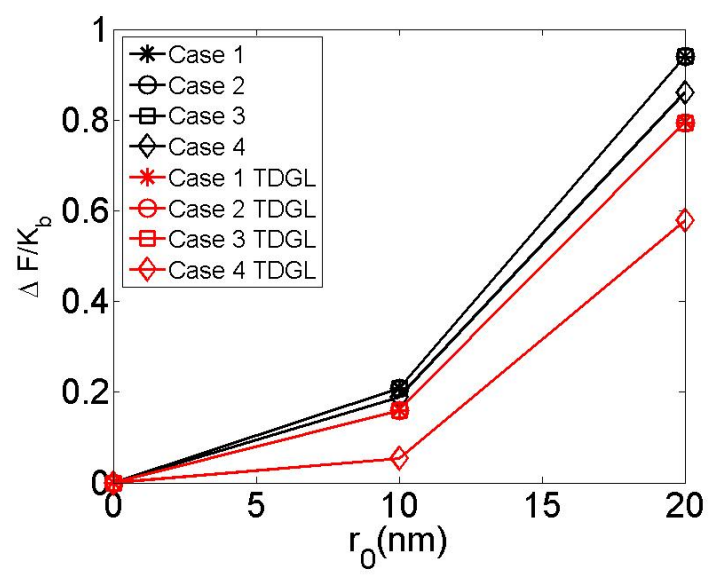

(a)

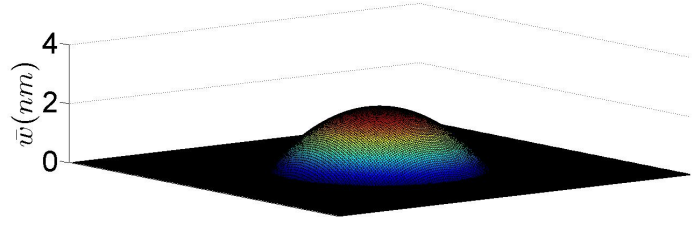

(b)

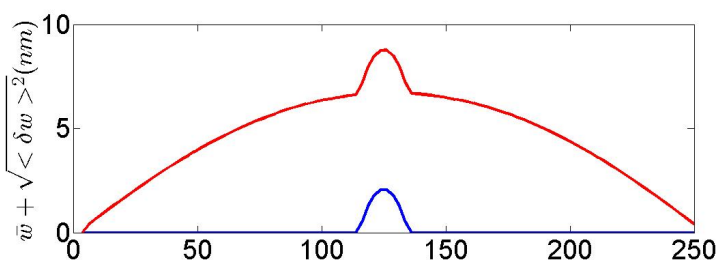

(c)

Figure 8: (a) Comparison of membrane free energy change as a function of $r_{0}$. Black markers are our result. Red marks are TGDL simulation data extracted from Figure 4 in [23]. (b) Three dimensional plot of equilibrium position $\bar{w}$ of a membrane with a region of non-zero spontaneous curvature $C_{0}=0.04 \mathrm{~nm}^{-1}, r_{0}=10 \mathrm{~nm}$, and zero-tension. We have just chosen the mid $40 \mathrm{~nm} \times 40 \mathrm{~nm}$ region to show for clarity. (c) Cross-sectional plot of a thermally fluctuating membrane with a region of non-zero spontaneous curvature in the middle of the square patch. Blue line is the equilibrium position. We have added the standard deviation $\sqrt{\left\langle\delta w^{2}\right\rangle}$ to the equilibrium position and plotted it as the red line.

\subsection{Heterogeneous membrane}

So far we have assumed that our membrane has the same bending modulus everywhere. We have reproduced some well known results on membrane fluctuations and have extended some other results, e.g. influence of boundary conditions on the magnitude of fluctuations. However, our computational method is capable of handling heterogeneous membranes in which the bending modulus and spontaneous curvature depend on position. This is the case for every cell membrane and can be produced in vesicles in which phase separation results in regions with different lipid compositions [48]. In order to demonstrate the capabilities of our method we will use it to extract some information from experimental data on the membranes of red blood cells infected with malarial parasites [35]. These experiments show that the fluctuations of the membrane are significantly influenced by localized membrane stiffening caused by the parasite. To model this situation we choose a square patch of membrane of side $250 \mathrm{~nm}$. The middle portion of this membrane of side $50 \mathrm{~nm}$ has a different bending modulus $K_{b 2}=100 k_{B} T$, while elsewhere $K_{b 1}=10 k_{B} T$. We compute the variance of the fluctuations in $w$ that are typically measured in experiments. Our goal is to extract a membrane tension by comparing our results with the experiment. The membrane is simply supporte on four sides and we apply a constant force per unit length on all edges. The results from this heterogeneous membrane model are shown in Figure 9. To match the experiments we had to use a membrane tension as $0.3 \mathrm{pN} / \mathrm{nm}$, which is less than the lytic tension of the membrane. In Figure 9a the computational result correctly estimates the magnitude of the fluctuations measured in [35]. In this figure red represents large fluctuations and blue corresponds to small fluctuations. The overall profile in Figure $9 \mathrm{~b}$ is different from a homogeneous membrane in section 2.4, where the fluctuation at the center point is 
larger than points near the boundary. In this heterogeneous membrane, due to large bending modulus that suppresses thermal fluctuation, the variance in the middle region is smaller. To see this effect clearly, one cross-sectional profile of the membrane variance is shown in Figure 9c. We measure the difference between the middle and the margin of the concave region marked as $\mathrm{A}$ and $\mathrm{B}$, respectively, and plot it as a function of membrane mid-region bending modulus as shown in Figure 9d. If the membrane tension is known then this kind of plot can be used to infer the difference in bending modulus between the stiffer and softer regions.

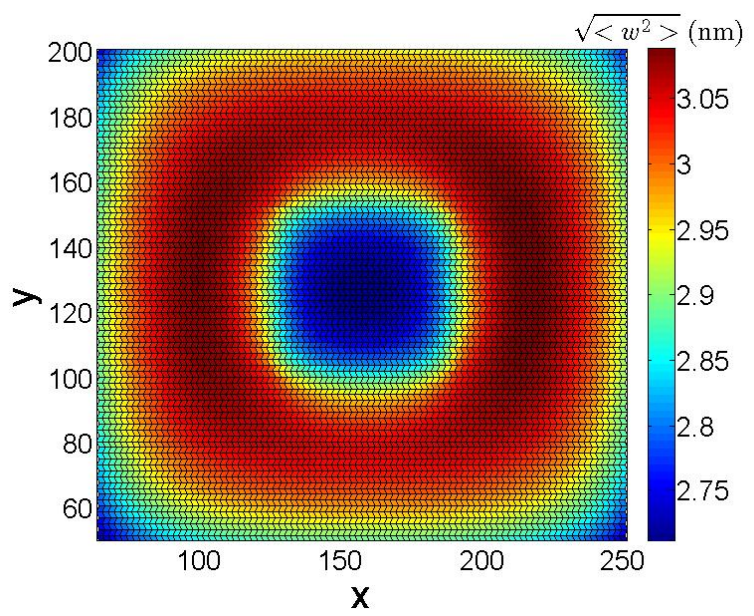

(a)

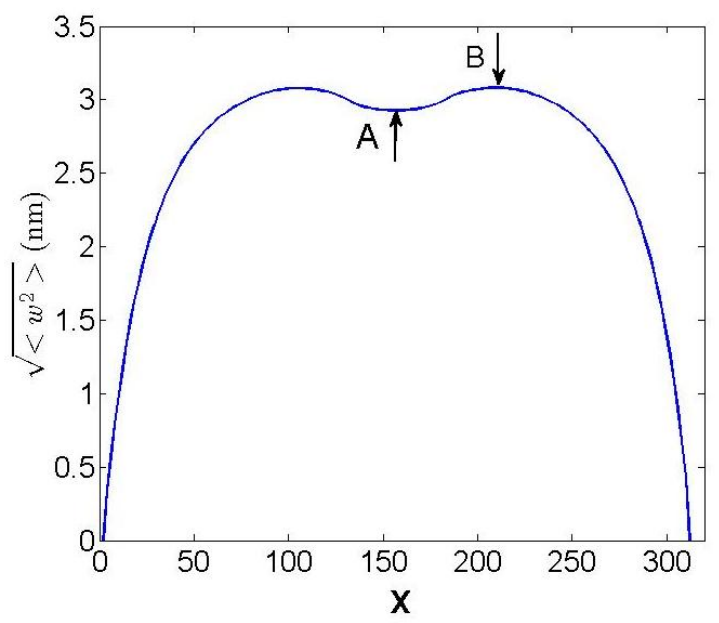

(c)

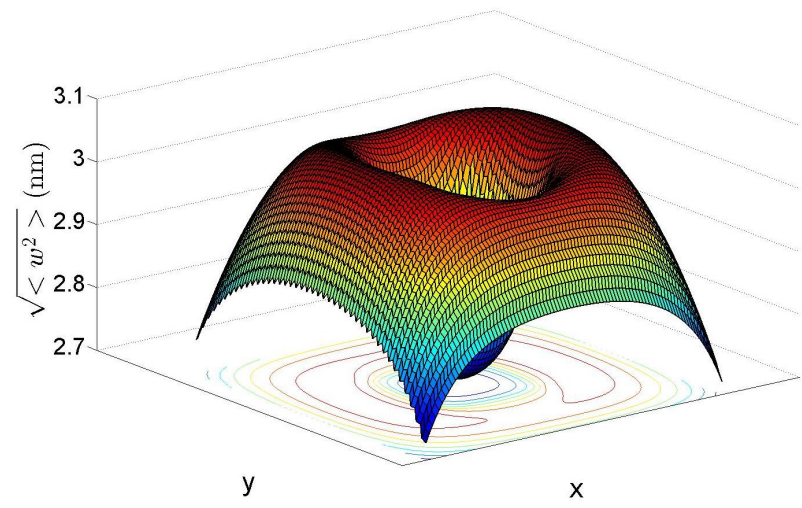

(b)

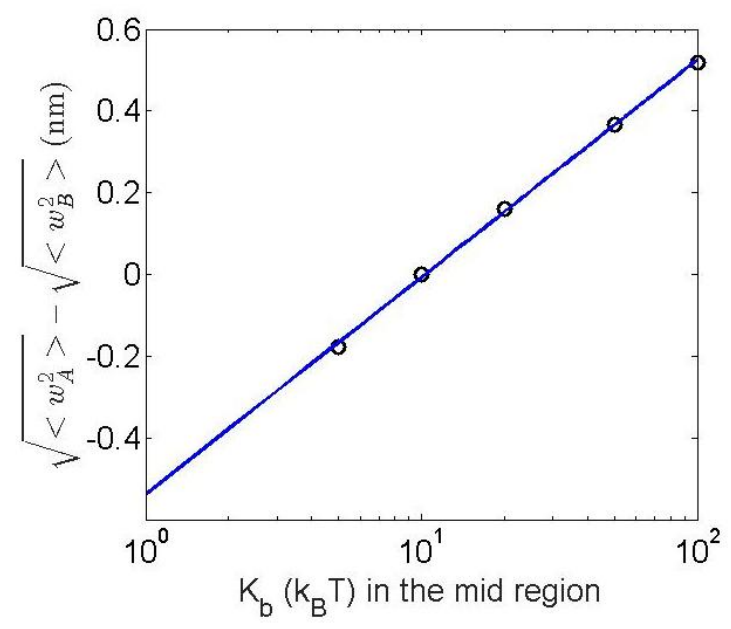

(d)

Figure 9: (a) Two dimensional contour plot of standard deviation in $w(x, y)$ of a heterogeneous membrane. (b) Three dimensional plot of standard deviation of the same heterogeneous membrane. (c) Cross-sectional profile of standard deviation. A and B are respectively the middle and margin of the concave region in the center. (d) Difference between the middle and the margin of the concave region versus membrane mid-region bending modulus. The bending modulus outside the mid-region of the membrane is $10 k_{B} T$ at $T=300 \mathrm{~K}$. The tension $F=0.3 \mathrm{pN} / \mathrm{nm}$ for these plots. 


\section{Cell model}

In this section we propose a different method to compute the relation between tension and projected area of a lipid membrane. We recall that a lipid membrane is a liquid in plane. The earliest (reasonably accurate) model for the partition function of a liquid was written by Lennard-Jones and Devonshire [36, 37]. The liquid was treated as a collection of cells with one molecule per cell. Each molecule could only move within its cell in a quadratic potential imposed on it from all other surrounding molecules. The partition function for the liquid $Z$ could then be written as $Z=z^{n}$ where $z$ was the partition function of a molecule in a single cell and $n$ was the number of molecules in the sample. Later authors generalized this model to allow for correlated motions of neighboring cells and also to include more than one molecule per cell [49]. Both three dimensional and two dimensional liquids were considered, but the possibility of out-of-plane deflections of two dimensional liquids was not considered. Our goal is to account for this energetic contribution in our calculation of the partition function of a lipid membrane. Note that the partition function of the whole membrane $Z$ can be written in terms of the partition function of a single cell $z$. This is reminiscent of the freely jointed chain model of polymers [28], whose partition function also is of the form $z^{n}$, where $n$ is the number of links.

To construct the partition function let us think of a lipid membrane as a collection of cells of radius $R_{0}$ with more than one lipid molecule per cell. Interestingly, a coarse grained simulation model of this type has already been built by Yuan et al. [50]. In this model each 'cell' interacts with its nearest neighbors through a potential that depends only on the distance (hence, direction independent) between their centers. This is similar to the assumption in the classic cellmodel for liquids [36, 37]. But, in the simulations of Yuan et al. [50] each cell can suffer some rotations and deflections relative to its nearest neighbors and these are penalized by an anisotropic (direction dependent) energy. This coarsegrained model has been shown to reproduce a large number of experimental results on vesicles and bi-layers [51, 52]. In some other work the cell-model has been used to study the membrane mediated interactions of proteins embedded in a lipid bi-layer. The protein sits at the center of a cell and the shape of the membrane surrounding the protein is computed starting from the Helfrich energy. The protein determines the boundary condition for the shape equation while its concentration in the membrane determines a length scale which sets the radius $R_{0}$ of the cell. If the tension is zero then the membrane shapes in the cell surrounding the protein are catenoids whose energy can be computed to complete the calculation of the partition function $z$ [53]. The number of cells $n$ can be determined from the concentration of the proteins and then $Z=z^{n}$ can be computed. However, we want to compute $Z$ in the absence of any protein, but in the presence of a constant tension $F$ in the membrane. Our calculation rests upon the idea that $\lambda=\sqrt{K_{b} / F}$ is a length scale and that $R_{0} / \lambda \approx O(1)$. The center of our circular cell moves out of plane in response to thermal kicks. For simplicity, we will assume that our circular cell deforms into an oblique cone in response to an impact delivered by a bombarding molecule from the surroundings, as shown in Appendix. If the cell is assumed to lie on the $\mathbf{e}_{1}-\mathbf{e}_{2}$ plane when it is flat, then the apex of the cone is allowed to displace in the $\mathbf{e}_{1}, \mathbf{e}_{2}, \mathbf{e}_{3}$ directions. 


\subsection{Energy of a single cell}

In order to compute the partition function in our cell model we are going to express the energy of a deformed cell as function of the displacement of its apex. We assume the total energy of a single cell is given by [38]

$$
E=E_{0}+\frac{1}{2} K_{c} r^{2}+E_{b}+E_{p}
$$

where $E_{0}$ is the minimum of the potential imposed by the surrounding molecules on the cell, $K_{c}$ is a force constant, $r$ the distance of the center of a cell from the equilibrium position at the minimum of the potential well, and $E_{b}, E_{p}$ are the bending energy and potential energy (due to applied tension) of the cell deformed into an oblique cone. $E_{0}$ and $K_{c}$ depend on $F$ since the minimum will change depending on the applied tension and we will assume later that the cell size depends on $F$. However, as a first approximation we let them be constants. This feature of our model is different from the original cell model in which the volume of the liquid was held fixed rather than the tension (or pressure in three dimensions) applied on it. We can evaluate the partition function of a single cell by integrating the Boltzmann factor based on this energy.

Using expressions of local curvature and surface area in the Appendix, we compute both the bending energy and potential energy due to applied tension $F$ for our oblique cone. For convenience, we define two parameters indicating two degrees of freedom of the cone apex as $x=h / R, y=\tan \alpha$, where $R, h$ and $\alpha$ are defined in Appendix. The bending energy is

$$
E_{b}=\iint \frac{K_{b} C^{2}}{2} \mathrm{~d} A=\frac{K_{b} h^{2}}{2 R^{2}} \ln \frac{h}{b} \int_{0}^{2 \pi} \mathrm{d} \beta\left(\frac{\sin ^{2} \beta}{(x y \cos \beta-1)^{2}}+\cos ^{2} \beta\right)^{2} \sqrt{1+x y \cos \beta+\frac{x^{2}\left(1+y^{2}\right)}{2}} .
$$

The potential energy due to the applied tension is

$$
E_{p}=\iint \frac{F}{2}\left(z_{, x}^{2}+z_{, y}^{2}\right) \mathrm{d} A=\frac{F h^{2}}{4} \int_{0}^{2 \pi} \mathrm{d} \beta\left(\frac{\cos ^{2} \beta}{(x y \cos \beta-1)^{2}}+\sin ^{2} \beta\right) \sqrt{1+x y \cos \beta+\frac{x^{2}\left(1+y^{2}\right)}{2}} .
$$

The integrals in Egns. (30) and (31) are difficult to evaluate, but it is possible to simplify them if we assume that the deflections are small. By assuming $x, y \ll 1$, we have

$$
E_{b} \approx \frac{\pi K_{b} h^{2}}{R^{2}} \ln \frac{h}{b}, \quad E_{p} \approx \frac{\pi F h^{2}}{2} .
$$

Combining bending energy and potential energy together, and defining a dimensionless variable $\bar{h}=h / b$, where $b$ is the size of lipid, the integral for the partition function of one cell becomes

$$
z=\int_{-\infty}^{\infty} \int_{0}^{\infty} \int_{0}^{2 \pi} \exp \left[-\frac{E_{0}+\frac{1}{2} K_{c} r^{2}+E_{b}+E_{p}}{k_{B} T}\right] r \mathrm{~d} \theta \mathrm{d} r \mathrm{~d} \bar{h} \approx \frac{2 \pi k_{B} T}{K_{c}} \sqrt{\frac{\pi}{a}} \exp \left(\frac{a}{2 e} e^{-2 k}\right) \exp \left(-\frac{E_{0}}{k_{B} T}\right),
$$


where

$$
a=\frac{\pi K_{b} b^{2}}{k_{B} T R^{2}}, k=\frac{c}{a}=\frac{F R^{2}}{2 K_{b}} .
$$

Here, the integration over $\bar{h}$ was approximated by the Laplace method.

\subsection{Number of cells}

Having computed $z$ we need $n$ to find $Z=z^{n}$. The number of cells $n$ will depend on the radius $R_{0}$ of a single cell. We claim that $R_{0}$ depends linearly on the length scale $\lambda=\sqrt{K_{b} / F}$ [54]. It was shown in [54] that when the center of a circular patch of membrane under tension $F$ is displaced out of plane by some height $h$ then the radius of the boundary layer around the point force in which bending energy is significant is of the order $\lambda$. We take $R_{0}=b+2 \lambda$. We introduce the lipid size $b$ in the expression for $R_{0}$ to ensure that there is at least one lipid in one cell when the membrane is flat like a liquid confined to two dimensions (which happens when $F \rightarrow \infty$ ) or when the liquid offers no bending resistance (which happens when $K_{b}=0$ ). Then, the number of cells is given by $n=A /\left(\pi R_{0}^{2}\right)$ where $A$ is the reference area of the membrane. Hence, the free energy of this fluctuating membrane is $G=-n k_{B} T \ln z$. The thermally reduced area of the membrane can now be computed as

$$
\Delta A=-\frac{\partial G}{\partial F}
$$

We computed this derivative numerically and compared the result with Eqn. (14) in Figure 10a. We find that key features of the tension-area relation are captured by our analogue, for membranes, of the freely jointed chain model for polymers. It is instructive to see this analytically by approximating $R_{0} \approx 2 \lambda$ for a reasonable range of $F$. In this approximation the number of cells is given by

$$
n=\frac{A}{\pi R_{0}^{2}}=\frac{A F}{4 \pi K_{b}}
$$

Hence, the free energy of our fluctuating membrane is

$$
G \approx \frac{A F k_{B} T}{8 \pi K_{b}}\left(\ln \frac{4 \pi^{2}\left(k_{B} T\right)^{3}}{K_{c} b^{2}}-\frac{2 E_{0}}{k_{B} T}-\ln F\right)=\frac{A F k_{B} T}{8 \pi K_{b}}(C-\ln F),
$$

where we have taken the $\exp \left(\frac{a}{2 e} e^{-2 k}\right) \approx 1$ in Eqn. (33) because $b$ is about $1 \mathrm{~nm}$ and is much smaller than $R$. Those terms that are independent of tension $F$ are reduced to one variable $C$. Therefore, the reduced area is

$$
\frac{\Delta A}{A}=-\frac{1}{A} \frac{\partial G}{\partial F}=\frac{k_{B} T}{8 \pi K_{b}}(\ln F+1-C)
$$


The logarithmic dependence of $\triangle A / A$ on $F$ and the linear dependence on $1 / K_{b}$ which are apparent in Helfrich's expression are captured by our cell model. Meanwhile, the entropy of the membrane is given by

$$
S=-\frac{\partial G}{\partial T}=n k_{B} T \frac{\partial \ln z}{\partial T}
$$

We also compare in Figure 10b the entropy change as a function of tension predicted by the cell model with Eqn. 16 derived from the Helfrich model[8]. Membrane properties are $L=1 \mathrm{~mm}$ and $K_{b}=10 k_{B} T$. The cell model fitting parameters are chosen to be $E_{0}=6 \mathrm{pNnm}$ and $K_{c}=8.5 \mathrm{pN} / \mathrm{nm}$. Our cell model matches Helfrich's result both for the thermally reduced area and entropy change.

We now point out how our cell model is different from the Helfrich model. First, the entropy obtained from the cell model is extensive because it scales with the system size $A / b^{2}$. The expression obtained from the Helfrich model satisfies this property (that entropy is extensive) only when the system size $A / b^{2}$ is very large. Second, the change in entropy $\Delta S=S\left(F_{1}, T\right)-S\left(F_{2}, T\right)$ depends on $T$ in the cell model, but is independent of $T$ in the Helfrich model. This will lead to different predictions for the heat capacity of the membrane which may be testable in experiments of the type performed in [55].

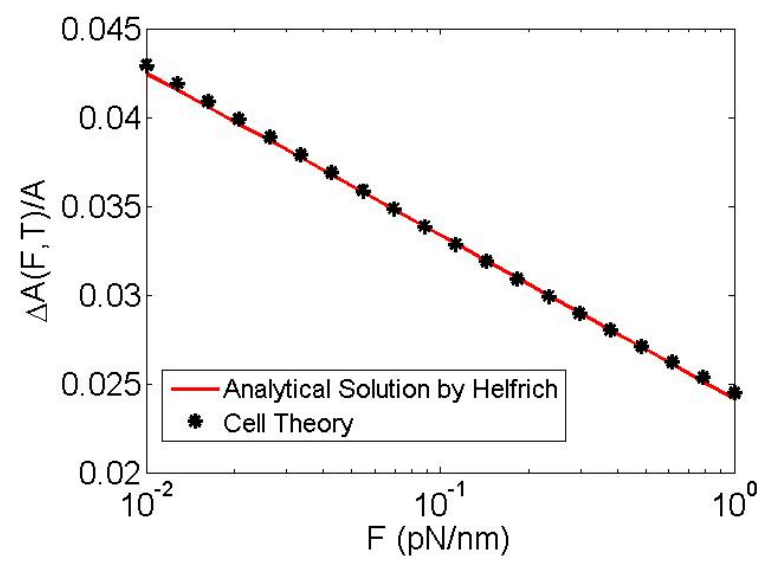

(a)

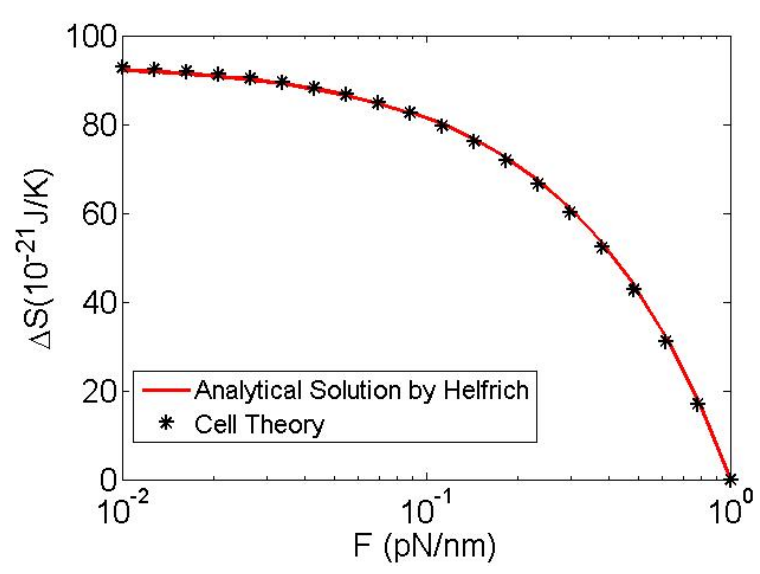

(b)

Figure 10: (a) Reduced area from our cell model compared with Helfrich's result. (b) Entropy change $\Delta S=S-\left.S\right|_{F=1}$ of the whole membrane compared with Helfrich's result. The temperature is $T=300 \mathrm{~K}$. Membrane properties are $L=1 \mathrm{~mm}$ and $K_{b}=10 k_{B} T$. The cell model fitting parameters are chosen to be $E_{0}=6 \mathrm{pNnm}$ and $K_{c}=8.5 \mathrm{pN} / \mathrm{nm}$.

\section{Conclusions}

In this paper we have proposed two new approaches to compute the partition function of a thermally fluctuating lipid membrane. Our first approach, based on the discretization of the membrane into equilateral triangles, deviates from many computational/simulation methods which start from a similar discretization, in that we compute the partition function using Gaussian integrals. Our method can accommodate any type of boundary condition and is not 
limited to homogeneous membranes. Furthermore, since it is based on triangular elements, a wealth of knowledge from the field of computational mechanics of shells can be brought to bear on membrane fluctuation problems through our techniques. An advantage of our method is that we can compute membrane free energies for only a modest computational cost; these are otherwise computationally intensive to calculate using Monte Carlo or TDGL methods. The disadvantages of our method are that we are restricted to (a) fluctuations small enough that there are no overhangs in the membrane (similar to the Monge gauge), and (b) quadratic Hamiltonians. On the other hand, Monte Carlo methods can handle large deformations including overhangs and self-contact, as well as non-quadratic Hamiltonians.

Our second approach, based on the cell model, is applicable only to homogeneous membranes. Our key insight in this model is that the cell size depends on the tension $F$. While our cell model cannot account for boundary conditions it may be fruitfully employed in situations where the out-of-plane deflections are constrained due to confinement. Earlier approaches based on normal modes run into difficulties in this situation because they do not clearly specify how the amplitudes of the modes are affected by the confinement. In the cell-model, on the other hand, we can account for the effect of confinement by prescribing the upper limit in the integral for the partition function $z$ as the maximum out-of-plane deflection allowed.

\section{Acknowledgements}

We acknowledge partial support for this work through an NSF CAREER award grant number NSF CMMI 09535448 .

\section{Appendix}

A circular cell deforms into an oblique cone when a molecule hits it at the center as indicated by the arrow. The geometrical parameters used in our calculation of the energy are described here.

In an oblique cone the line joining the apex to the center of the base is not perpendicular to the base. We indicate this by an angle $\alpha$ as shown in the Figure .11. If the height of the cone is $h$ and the radius of the base is $R$, let us try to compute the curvature at point $P$ denoted by combination of parameters $(x, y)$ or $(z, \beta)$. From geometry (see Figure $.11)$, the equation linking $(x, y)$ and $(z, \beta)$ is

$$
\begin{aligned}
& x=z \tan \alpha+\frac{(h-z) R \cos \beta}{h}=z \tan \alpha+r \cos \beta, \\
& y=\frac{(h-z) R \sin \beta}{h}=r \sin \beta,
\end{aligned}
$$

where $r=R(h-z) / h$ is the radius of the circular cross section of the oblique cone on which $P$ is located. From Eqn. 


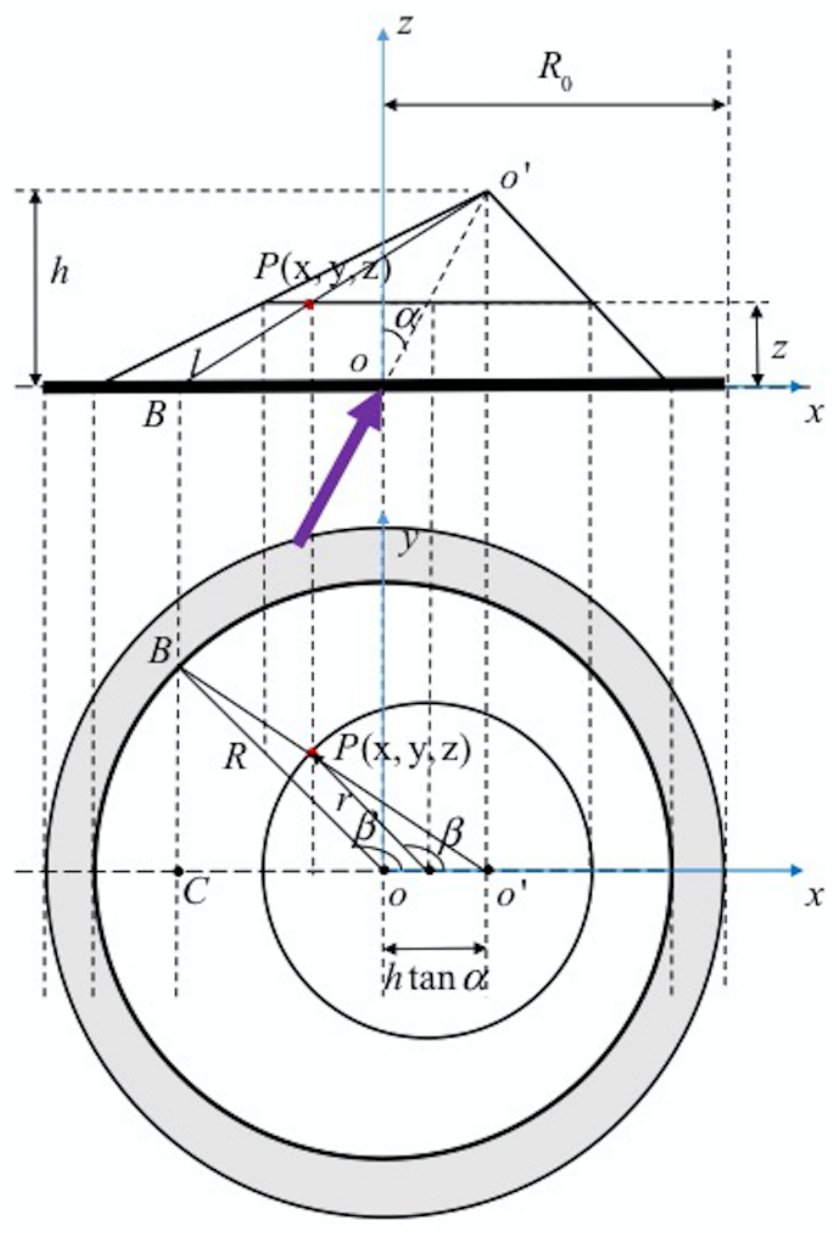

Figure .11: Oblique cone.

(.1) we get

$$
\begin{aligned}
(x-z \tan \alpha)^{2}+y^{2}=r^{2} & =\left(\frac{h-z}{h}\right)^{2} R^{2}, \\
\beta & =\arctan \frac{y}{x} .
\end{aligned}
$$

Thus, the differential relation between $z$ and $(x, y)$ is

$$
\begin{aligned}
\frac{\partial^{2} z}{\partial x^{2}} & =\frac{h^{2} \sin ^{2} \beta}{(h \cos \beta \tan \alpha-R)^{2}(h-z)}, \\
\frac{\partial^{2} z}{\partial y^{2}} & =\frac{h^{2} \cos ^{2} \beta}{R^{2}(h-z)} .
\end{aligned}
$$


Hence, the curvature at point $P$ is given by

$$
C=\frac{\left(1+z_{, x}^{2}\right) z_{, y y}+\left(1+z_{, y}^{2}\right) z_{, x x}-2 z, x z, y z, x y}{\left(1+z_{, x}^{2}+z_{, y}^{2}\right)^{3 / 2}} \cong z_{, x x}+z_{, y y}=\frac{h^{2} \sin ^{2} \beta}{(h \cos \beta \tan \alpha-R)^{2}(h-z)}+\frac{h^{2} \cos ^{2} \beta}{R^{2}(h-z)} .
$$

Note that when $\alpha=0$, the oblique cone becomes a right circular cone and the curvature reduces to

$$
C=\frac{h^{2} \sin ^{2} \beta}{R^{2}(h-z)}+\frac{h^{2} \cos ^{2} \beta}{R^{2}(h-z)}=\frac{h^{2}}{R^{2}(h-z)} .
$$

Let us compute the surface area of the oblique cone shown in the Figure .11 by making slices parallel to the base. The meridian $l$ depends on the angle $\beta$ in a complicated way as

$$
\begin{aligned}
l^{2} & =h^{2}+O^{\prime} C^{2}+B C^{2} \\
l & =\sqrt{h^{2} \sec ^{2} \alpha+R^{2}+2 h R \cos \beta \tan \alpha} .
\end{aligned}
$$

Since we write the curvature in terms of $(z, \beta)$, we also want the surface area as a function of $(z, \beta)$ instead of $(l, \beta)$. The integral for the total surface area is

$$
\begin{aligned}
A & =\int_{0}^{h} \mathrm{~d} z \int_{0}^{2 \pi} \mathrm{d} \beta \frac{R(h-z)}{h} \sqrt{\sec ^{2} \alpha+\left(\frac{R}{h}\right)^{2}+\frac{2 R}{h} \cos \beta \tan \alpha} \\
& =\frac{R^{2}}{2} \int_{0}^{2 \pi} \mathrm{d} \beta \sqrt{1+\frac{h^{2}}{R^{2}}\left(1+\tan ^{2} \alpha\right)+\frac{2 h}{R} \cos \beta \tan \alpha}
\end{aligned}
$$

Note again that when $\alpha=0$, the oblique cone becomes a right circular cone and the surface area reduces to exactly what we expect:

$$
A=\frac{R^{2}}{h^{2}} \int_{0}^{h}(h-z) \mathrm{d} z \int_{0}^{2 \pi} \mathrm{d} \beta \sqrt{1+\left(\frac{h^{2}}{R}\right)^{2}}=\pi R \sqrt{R^{2}+h^{2}}
$$

\section{Reference}

[1] Helfrich, W. (1973). Elastic properties of lipid bilayers: theory and possible experiments. Z. Naturforsch. c, 28(11), 693-703.

[2] Weeks, J. D. (1977). Structure and thermodynamics of the liquidvapor interface. The Journal of Chemical Physics, 67(7), 3106-3121.

[3] Fasolino, A., Los, J. H., Katsnelson, M. I. (2007). Intrinsic ripples in graphene. Nature materials, 6(11), 858-861.

[4] Rodriguez-Garcia, R., Mell, M., Lopez-Montero, I., Netzel, J., Hellweg, T. and Monroy, F. (2011), Polymersomes: smart vesicles of tunable rigidity and permeability, Soft Matter 7(4), 1532-1542.

[5] Evans, E., Rawicz, W. (1990). Entropy-driven tension and bending elasticity in condensed-fluid membranes. Physical Review Letters, 64(17), 2094.

[6] Helfrich, W. (1975). Out-of-plane fluctuations of lipid bilayers. Zeitschrift fr Naturforschung. Section C: Biosciences, $30(6), 841$.

[7] Milner, S. T., Safran, S. A. (1987). Dynamical fluctuations of droplet microemulsions and vesicles. Physical Review A, 36(9), 4371.

[8] Boal, D., Boal, D. H. (2012). Mechanics of the Cell. Cambridge University Press.

[9] Helfrich, W. (1985). Effect of thermal undulations on the rigidity of fluid membranes and interfaces. Journal de Physique, 46(7), 1263-1268. 
[10] Hanlumyuang, Y., Liu, L., Sharma, P. (2014). Revisiting the entropic force between fluctuating biological membranes. Journal of the Mechanics and Physics of Solids, 63, 179-186.

[11] Paulose, J., Vliegenthart, G. A., Gompper, G., Nelson, D. R. (2012). Fluctuating shells under pressure. Proceedings of the National Academy of Sciences, 109(48), 19551-19556.

[12] Nelson, D. R., Peliti, L. (1987). Fluctuations in membranes with crystalline and hexatic order. Journal de physique, 48(7), 1085-1092.

[13] Auth, T., Gompper, G. (2013). Fluctuation pressure of biomembranes in planar confinement. Physical Review E, 88(1), 010701.

[14] Ramakrishnan, N., Kumar, P. S., Ipsen, J. H. (2010). Monte Carlo simulations of fluid vesicles with in-plane orientational ordering. Physical Review E, 81(4), 041922.

[15] Tourdot, R. W., Ramakrishnan, N., Radhakrishnan, R. (2014). Defining the free-energy landscape of curvature-inducing proteins on membrane bilayers. Physical Review E, 90(2), 022717.

[16] Lin, L. C. L., Gov, N., Brown, F. L. (2006). Nonequilibrium membrane fluctuations driven by active proteins. The Journal of chemical physics, 124(7), 074903

[17] Zhang, T., Li, X., Kadkhodaei, S., Gao, H. (2012). Flaw insensitive fracture in nanocrystalline graphene. Nano letters, 12(9), 4605-4610

[18] Zhang, T., Li, X., Gao, H. (2014). Defects controlled wrinkling and topological design in graphene. Journal of the Mechanics and Physics of Solids, 67, 2-13

[19] Harmandaris, V. A., Deserno, M. (2006). A novel method for measuring the bending rigidity of model lipid membranes by simulating tethers. The Journal of chemical physics, 125(20), 204905.

[20] Hanlumyuang, Y., Liu, L., Sharma, P. (2014). Revisiting the entropic force between fluctuating biological membranes. Journal of the Mechanics and Physics of Solids, 63, 179-186.

[21] Ramakrishnan, N., Kumar, P. S., Radhakrishnan, R. (2014). Mesoscale computational studies of membrane bilayer remodeling by curvatureinducing proteins. Physics reports, 543(1), 1-60.

[22] David, F., Nelson, D., Piran, T., Weinberg, S. (1989). Statistical mechanics of membranes and surfaces. Nelson, T. Piran and S. Wein.

[23] Agrawal, N. J., Radhakrishnan, R. (2009). Calculation of free energies in fluid membranes subject to heterogeneous curvature fields. Physical Review E, 80(1), 011925.

[24] Lin, L. C. L., Brown, F. L. (2004). Brownian dynamics in Fourier space: membrane simulations over long length and time scales. Physical review letters, 93(25), 256001.

[25] Hughes T. J. R. (2000). The Finite Element Method, Dover Publications.

[26] Gompper, G., Kroll, D. M. (1996). Random surface discretizations and the renormalization of the bending rigidity. Journal de Physique I, 6(10), 1305-1320.

[27] Nelson, D. (2004). Statistical mechanics of membranes and surfaces. World Scientific.

[28] Weiner J. H. (2002). Statistical Mechanics of Elasticity, Dover Publications.

[29] Landau L. D. and Lifshitz E. M. (1980). Statistical Physics, Butterworth Heinemann.

[30] Zhang, Y., Crothers, D. M. (2003). Statistical mechanics of sequence-dependent circular DNA and its application for DNA cyclization. Biophysical journal, 84(1), 136-153.

[31] Su, T., Purohit, P. K. (2010). Thermomechanics of a heterogeneous fluctuating chain. Journal of the Mechanics and Physics of Solids, 58(2), 164-186.

[32] Su, T., Purohit, P. K. (2011). Fluctuating elastic filaments under distributed loads. Mol Cell Biomech, 8, $215-232$.

[33] Su, T., Purohit, P. K. (2012). Semiflexible filament networks viewed as fluctuating beam-frames. Soft Matter, 8(17), 4664-4674.

[34] Flory, P. J., Volkenstein, M. (1969), Statistical mechanics of chain molecules. Biopolymers, 8: 699700.

[35] Park, Y., Diez-Silva, M., Popescu, G., Lykotrafitis, G., Choi, W., Feld, M. S., Suresh, S. (2008). Refractive index maps and membrane dynamics of human red blood cells parasitized by Plasmodium falciparum. Proceedings of the National Academy of Sciences, 105(37), 13730-13735.

[36] Lennard-Jones, J. E., Devonshire, A. F. (1937). Critical phenomena in gases. I. Proceedings of the Royal Society of London. Series A, 
Mathematical and Physical Sciences, 53-70.

[37] Lennard-Jones, J. E., Devonshire, A. F. (1938). Critical phenomena in gases. II. Vapour pressures and boiling points. Proceedings of the Royal Society of London. Series A, Mathematical and Physical Sciences, 1-11.

[38] Henchman, R. H. (2003). Partition function for a simple liquid using cell theory parametrized by computer simulation. The Journal of chemical physics, 119(1), 400-406

[39] BenShaul, A., Szleifer, I., Gelbart, W. M. (1985). Chain organization and thermodynamics in micelles and bilayers. I. Theory. The Journal of chemical physics, 83(7), 3597-3611.

[40] May, S., Ben-Shaul, A. (1999). Molecular theory of lipid-protein interaction and the L -H II transition. Biophysical journal, 76(2), 751-767.

[41] May, S., Harries, D., Ben-Shaul, A. (2000). Lipid demixing and protein-protein interactions in the adsorption of charged proteins on mixed membranes. Biophysical journal, 79(4), 1747-1760.

[42] Mbamala, E. C., Ben-Shaul, A., May, S. (2005). Domain formation induced by the adsorption of charged proteins on mixed lipid membranes. Biophysical journal, 88(3), 1702-1714.

[43] Freund, L. B. (2013). Entropic pressure between biomembranes in a periodic stack due to thermal fluctuations. Proceedings of the National Academy of Sciences, 110(6), 2047-2051.

[44] Audoly, B. , Pomeau, Y. (2010). Elasticity and Geometry, Oxford University Press .

[45] Abad, J., Asorey, M., Cruz, A. (Eds.). (1986). New perspectives in quantum field theories: proceedings, World Scientific.

[46] Fraternali, F., Marcelli, G. (2012). A multiscale approach to the elastic moduli of biomembrane networks. Biomechanics and modeling in mechanobiology, 11(7), 1097-1108.

[47] Zhang, Y., Crothers, D. M. (2003). Statistical mechanics of sequence-dependent circular DNA and its application for DNA cyclization. Biophysical journal, 84(1), 136-153.

[48] Baumgat, T., Hess, S. T. and Webb, W. W. (2003). Imaging coexisting fluid domains in biomembrane models coupling curvature and line tension. Nature, $425,821-824$.

[49] De Boer, J. (1954). Cell cluster theory for the liquid state, Physica, 20, 655-664..

[50] Yuan, H., Huang, C., Li, J., Lykotrafitis, G., Zhang, S. (2010). One-particle-thick, solvent-free, coarse-grained model for biological and biomimetic fluid membranes. Physical Review E, 82(1), 011905.

[51] Yuan, H., Huang, C., Zhang, S. (2010). Virus-inspired design principles of nanoparticle-based bioagents. PloS one, 5(10), e13495.

[52] Yuan, H., Huang, C., Zhang, S. (2010). Dynamic shape transformations of fluid vesicles. Soft Matter, 6(18), $4571-4579$.

[53] Huang, C., Yuan, H., Zhang, S. (2011). Coupled vesicle morphogenesis and domain organization. Applied Physics Letters, 98(4), 043702.

[54] Powers, T. R., Huber, G., Goldstein, R. E. (2002). Fluid-membrane tethers: minimal surfaces and elastic boundary layers. Physical Review $E, 65(4), 041901$

[55] Marsh, D. (2012). Thermodynamics of phospholipid self-assembly. Biophysical journal, 102(5), 1079-1087. 